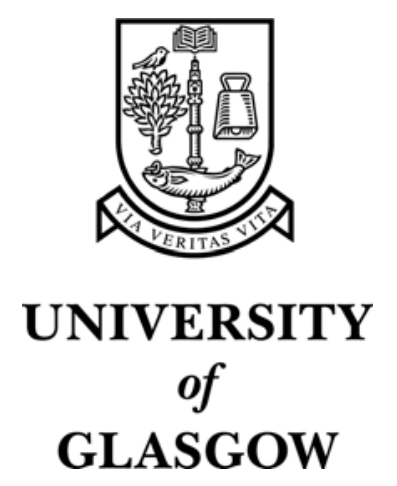

Shimojima, M. and Miyazawa, T. and Ikeda, Y. and McMonagle, E.L. and Haining, H. and Akashi, H. and Takeuchi, Y. and Hosie, M.J. and Willett, B.J. (2004) Use of CD134 As a Primary Receptor by the Feline Immunodeficiency Virus. Science 303(20):pp. 1192-1195.

http://eprints.gla.ac.uk/3145/ 
This is the author's version of the work. It was posted here by permission of the AAAS for personal use not for redistribution. The definitive version was published in SCIENCE (Vol. 303 Feb. 2004),

doi : http://dx.doi.org/10.1126/science.1092124 


\title{
Use of CD134 as a primary receptor by the feline immunodeficiency virus
}

\author{
Masayuki Shimojima $^{{ }^{*}}$, Takayuki Miyazawa ${ }^{2,3^{*}}$, Yasuhiro Ikeda ${ }^{4}$, Elizabeth L. \\ McMonagle $^{5}$, Hayley Haining ${ }^{5}$, Hiroomi Akashi ${ }^{1}$, Yasuhiro Takeuchi ${ }^{4}$, Margaret J. \\ Hosie $^{5}$ \& Brian J. Willett ${ }^{5 \dagger}$ \\ ${ }^{1}$ Department of Veterinary Microbiology, Graduate School of Agricultural and \\ Life Sciences, The University of Tokyo, 1-1-1 Yayoi, Bunkyo-ku, Tokyo \\ 113-8657, Japan \\ ${ }^{2}$ Department of Veterinary Public Health, Obihiro University of Agriculture and Veterinary \\ Medicine, Inada-cho, Obihiro, Hokkaido 080-8555, Japan \\ ${ }^{3}$ Host and Defense, PRESTO, Japan Science and Technology Agency, 4-1-8 Honcho, \\ Kawaguchi, Saitama 332-0012, Japan \\ ${ }^{4}$ Department of Immunology and Molecular Pathology, Windeyer Institute of Medical \\ Sciences, University College London, London W1T 4JF, UK \\ ${ }^{5}$ Department of Veterinary Pathology, University of Glasgow, Glasgow G61 1QH, UK.
}

*These authors contributed equally to this work

${ }^{\dagger}$ To whom correspondence should be addressed. E-mail: b.willett@vet.gla.ac.uk 
Feline immunodeficiency virus (FIV) induces a disease similar to AIDS in cats, yet in contrast to $\mathrm{HIV}$, CD4 is not the viral receptor. Here, we identify a primary receptor for FIV as CD134 (OX40), a T cell activation antigen and co-stimulatory molecule. CD134 expression promotes viral binding and renders cells permissive for viral entry, productive infection and syncytium formation. Infection is CXCR4-dependent, analogous to infection with X4 strains of HIV. Thus, despite the evolutionary divergence of the feline and human lentiviruses, both viruses utilise receptors that target the virus to a subset of cells pivotal to the acquired immune response. 
The primary event in the process of viral entry into a target cell is the interaction between the virus and its cellular receptor and the specificity of this interaction determines both viral cell tropism and pathogenicity. For the primate lentiviruses the viral receptor is CD4, targeting the virus to helper T cells, resulting in their depletion and the eventual development of AIDS (1). However, CD4-expression alone is insufficient to confer susceptibility to infection with HIV, which also depends on expression of co-receptors, principally the chemokine receptors CXCR4 and CCR5 (2). The virus attaches via a high affinity interaction with CD4, resulting in a conformational change in the envelope glycoprotein (Env) and exposing the binding site for the chemokine receptor (3). This then triggers a further conformational change that exposes the fusion domain of the viral transmembrane protein gp41 and enables fusion of the viral and cellular membranes (4).

The feline immunodeficiency virus (FIV) is unique among the non-primate lentiviruses in that in its natural host species, the domestic cat, it induces a disease similar to AIDS, characterised by a progressive depletion of CD4+ T lymphocytes (5). Consequently, an immunodeficiency syndrome develops characterised by wasting, neurological manifestations, chronic stomatitis and gingivitis and an increased incidence of lymphoma (6). However, CD4 is not the primary receptor for FIV, suggesting that the virus may bind to an alternative molecule in order to target CD4+ T cells (7). Partial elucidation of the mechanism of infection with FIV was provided by the discovery that primary isolates and laboratory strains of virus use CXCR4 as a co-factor for infection $(8,9)$. However CXCR4 expression alone was insufficient to confer susceptibility to infection with primary isolates of FIV, indicating the existence of an as yet unidentified primary receptor for the virus. 
To identify the primary receptor for FIV, we generated a cDNA library in the pMX retroviral vector from an IL2-dependent CD4+ feline T cell line (MYA-1), which is highly susceptible to infection with FIV (10). Following transduction of P3U1 murine myeloma cells with the library, cells capable of binding FIV were identified by “panning” with FIV-coated plates (11). Positive colonies were expanded and genomic DNA prepared and the cDNA insert amplified from the retroviral vector by PCR. Of three cDNAs cloned from the library, only one showed reproducible binding to FIV when re-expressed in P3U1 cells and was characterised as the feline homologue of CD134 (DDBJ accession no. AB128982). CD134 was first described as MRC OX-40, an antigen expressed on activated rat CD4+ T lymphocytes and a novel member of the TNF-NGF receptor family $(12,13)$. The feline CD134 cDNA identified here predicts a 270 amino acid protein with strong identity to human CD134 (Fig. 1A). To confirm that the feline CD134 protein binds FIV, we expressed the cDNA in the human cell line Plat-E and the murine cell line NIH-3T3. Specific FIV binding was detected on Plat-E and NIH 3T3 cells transduced with CD134 but not with cells expressing CD2 from the same vector (Fig. 1B.).

To assess whether CD134 acts as a functional viral receptor, the feline large granular lymphoma cell line MCC (these cells are refractory to infection with primary strains of FIV) was stably transduced with a retroviral vector bearing the CD134 cDNA. Surface CD134 expression was detected by flow cytometry (Fig. 2A) while a $\sim 45 \mathrm{kDa}$ species was detected by immunoblotting (Fig. 2B). CD134-expression rendered MCC cells permissive for infection with the five primary strains of FIV tested (Fig. 2C) while cells transduced with vector only remained non-permissive. To quantify viral entry into the CD134-expressing cells, we prepared HIV pseudotypes bearing FIV Env proteins and carrying a luciferase gene. Each successful entry event 
would trigger luciferase production in the target cells. MCC-CD134 cells were permissive for infection with pseudotypes bearing the Env proteins of either the GL8 or TM2 strains of FIV (Fig. 2D.), confirming that CD134 expression overcame the block to viral entry into these cells. CD134-expression also relieved the block to infection in the feline fibroblast line AH927 while a control vector encoding the FeLV-C receptor (FeLVC-R) had no effect (Fig.S1).

Syncytium formation occurs when Env expressed on the surface of a cell comes into contact with an adjacent cell expressing the viral receptor and co-receptor (4). Thus, transfection of cells expressing the viral receptor and co-receptor with envexpression vectors should trigger syncytium formation. Accordingly, transfection of AH927-CD134 cells with constructs bearing the env genes from primary isolates (Fig. 3E-G) led to the development of syncytia whereas no syncytia were observed in the control cultures (Fig. 3A-C) expressing the FeLV-CR. In contrast, FIV-PET env (derived from a FIV strain analogous to CD4-independent strains of HIV (14)) induced syncytium formation in both the CD134 (Fig. 3H) and FeLV-CR-expressing (Fig. 3D) cells, consistent with this Env interacting with CXCR4 in the absence of a primary receptor (15).

Human cells are refractory to infection with primary strains of FIV (16). We therefore examined species-specificity of the interaction between FIV and CD134. The human cell line HeLa was stably transduced with either feline or human CD134 and surface expression of CD134 was confirmed by flow cytometry (Fig. 4B). Although ectopic expression of feline CD134 rendered HeLa cells permissive for infection with primary FIV strain GL8 or TM2 pseudotypes (Fig. 4A) human CD134 expression had little effect. In contrast, the susceptibility of the feline and human CD134-expressing cells to HIV pseudotypes with VSV-G envelope was broadly similar to the control 
HeLa cells. Similarly, feline, but not human, CD134 expression permitted infection with HIV pseudotypes bearing FIV envelopes and encoding GFP (Fig. 4B). These results suggest that the specificity of the interaction between the virus and its primary receptor may be a major determinant of the species specificity of FIV.

Since infection with both primary and cell culture adapted strains of FIV is CXCR4-dependent $(8,9)$, we determined whether CD134-dependent infection required co-expression of CXCR4. HeLa cells express high levels of CXCR4 and human CXCR4 is an efficient co-receptor for FIV (17). Infection of HeLa-CD134 cells with GL8 and TM2 pseudotypes was inhibited efficiently by the selective CXCR4 antagonist AMD3100 while infection with control HIV (VSV) pseudotypes was not affected (Fig. 4C), confirming an absolute requirement for both receptor and coreceptor.

Further evidence for a role for CD134 in viral infection was provided by downregulation of CD134 from the surface of FIV infected cells (Fig. S2). Moreover, CD134-expressing cells expressed similar levels of CXCR4 to control cells, indicating that CD134 did not mediate its effect on FIV infection by modulating CXCR4 expression (Fig. S3).

We have shown that CD134 functions as a novel primary receptor for an immunodeficiency-causing lentivirus. CD134 expression is largely restricted to CD4+ T lymphocytes (12,18-20), however, in humans and mice CD134 is also expressed at lower levels on activated CD8+ T cells $(18,21)$, macrophages and activated B cells (22). CD4+ T cells are the primary target for FIV in early infection, while in chronic infection CD8+ T cells and B cells are also infected $(23,24)$, the tropism of FIV in vivo is consistent with the predicted expression of CD134. In addition, the viral coreceptor CXCR4 is expressed widely in the cat (activated T cells, B cells and 
monocytes (25)) and since some primary and cell culture adapted strains of FIV can infect via CXCR4 alone (26) (CD134-independent infection), the broadening of cell tropism of the virus in chronic infection may represent a shift towards CD134independent infection.

Signalling through CD134 plays a crucial role in the survival and proliferation of CD4+ T cells that have encountered antigen (20). By targeting CD134-expressing cells, FIV would selectively deplete a subset of CD4+ T cells that is integral to the development of antigen-specific $\mathrm{T}$ cell responses. In contrast, by utilising CD4 as a primary receptor, HIV has the potential to infect all CD4+ T cells and induce a more profound immune defect. However, the cell tropism of HIV is restricted by the expression of the viral co-receptor which, for the majority of strains that are transmitted, is CCR5. CCR5 expression on CD4+ T cells is restricted to an effector / memory $\mathrm{T}$ cell subset $(27,28)$. Thus despite the use of distinct primary binding receptors, both the human and feline viruses selectively impair antigen-specific helper T cell responses.

Vaccination may lead to enhancement of infection in the feline model of AIDS (29). Our new data may shed new light on the mechanism of enhancement, since CD134 is a T cell activation antigen with expression in vivo restricted predominately to CD4+ T cells. Vaccination may induce an expansion of a population of cells expressing the viral receptor so that if sterilising immunity is not achieved, vaccination may prove counter-productive.

That two lentiviruses with host species as divergent as human beings and the domestic cat should use distinct primary receptors to target the similar T cell subsets underlines the central role of CD4+ T lymphocyte infection in the pathogenesis of AIDS. Whether the feline and human lentiviruses evolved from a common ancestor, 
such as a CD4 or CD134-independent virus, is an intriguing question for the development of viral virulence, and this study represents the first step towards providing a solution. 
References and notes

1. Q. J. Sattentau and R. A. Weiss, Cell 52,631 (1988).

2. E. A. Berger, P. M. Murphy, J. M. Farber, Annual Review of Immunology 17,657 (1999).

3. P. D. Kwong et al., Nature 393,648 (1998).

4. R. W. Doms and J. P. Moore, J.Cell Biol. 151,F9 (2000).

5. N. C. Pedersen, E. W. Ho, M. L. Brown, J. K. Yamamoto, Science 235,790 (1987).

6. N. C. Pedersen, in The Retroviridae, J. A. Levy, Ed. (Plenum Press, New York, 1993), vol. 2 ,chap. 3.

7. A. deParseval and J. H. Elder, J.Virol. 75,4528 (2001).

8. B. J. Willett, M. J. Hosie, J. C. Neil, J. D. Turner, J. A. Hoxie, Nature 385,587 (1997).

9. J. Richardson et al., J.Virol. 73,3661 (1999).

10. T. M. Miyazawa et al., Arch.Virol. 108,131 (1989).

11. M. Shimojima et al., Anal.Biochem. 315,138 (2003).

12. D. J. Paterson et al., Mol.Immunol. 24,1281 (1987).

13. S. Mallett, S. Fossum, A. N. Barclay, EMBO J. 9,1063 (1990).

14. M. J. Endres et al., Cell 87,745 (1996).

15. M. J. Hosie et al., J.Virol. 72,2097 (1998).

16. B. J. Willett et al., J.Virol. 71,6407 (1997).

17. B. J. Willett et al., J.Virol. 72,6475 (1998).

18. A. Al Shamkhani et al., Eur.J.Immunol. 26,1695 (1996).

19. E. Stuber and W. Strober, J.Exp.Med. 183,979 (1996).

20. I. Gramaglia, A. D. Weinberg, M. Lemon, M. Croft, J.Immunol. 161,6510 (1998).

21. P. R. Baum et al., EMBO J. 13,3992 (1994).

22. H. Durkop, U. Latza, P. Himmelreich, H. Stein, Br.J.Haematol. 91,927 (1995). 
23. R. V. English, C. M. Johnson, D. H. Gebhard, M. B. Tompkins, J.Virol. 67,5175 (1993).

24. G. A. Dean, G. H. Reubel, P. F. Moore, N. C. Pedersen, J.Virol. 70,5165 (1996).

25. B. J. Willett, C. A. Cannon, M. J. Hosie, J.Virol. 77,709 (2003).

26. D. L. Lerner and J. H. Elder, J.Virol. 74,1854 (2000).

27. C. C. Bleul, L. J. Wu, J. A. Hoxie, T. A. Springer, C. R. Mackay, Proc.Natl.Acad.Sci.USA 94,1925 (1997).

28. L. J. Wu et al., J.Exp.Med. 185,1681 (1997).

29. M. J. Hosie, R. Osborne, G. Reid, J. C. Neil, O. Jarrett, Vet.Immunol.Immunopathol. 35,191 (1992).

30. This study was supported by the Japanese Ministry of Education, Culture, Sports,

Science and Technology and PRESTO, Japan Science and Technology Agency (T.M.)., NIH grant RO1 AI49765-01A1 (E.M., M.J.H. \& B.J.W.), The Wellcome Trust (M.J.H.\& H.H.), Japan Society for the Promotion of Science (M.S.), Medical Research Council (Y.T.) and Cancer Research UK (Y.I.). We are grateful to R.A. Weiss for critical evaluation of the manuscript and A.N. Barclay for the provision of antisera and N. Ishii for the human OX40 cDNA. 


\section{Figure legends}

Fig. 1. Molecular cloning and characterisation of feline CD134. (A) Alignment of feline and human CD134. Homologous amino acids are shaded light, identical residues are shaded dark. TM, transmembrane region. (B) Binding of FIV to CD134 expressing cells. Cell cultures transduced with CD134 or CD2 were incubated with culture supernatant from FIV-infected (thick line) or uninfected (thin line) cells followed by FIV Env detection by flow cytometry.

Fig. 2. CD134 expression renders feline cells permissive to infection with FIV. (A) Flow cytometric analysis of CD134 expression on MCC cells. Control (solid histogram) or anti-CD134 (Ber-ACT35, open histogram). Percentage positive is shown. (B) Immunoblot analysis with rabbit anti-rat OX40. MCC cells transduced with vector only (CON) were compared with cells transduced with the CD134 expression vector. A $\sim 45 \mathrm{kDa}$ species was detected in CD134-transduced cells (arrowed). (C) fCD134 expression renders MCC cells permissive of productive infection with primary strains of FIV. MCC-CON or MCC-CD134 were infected with the GL8, F0425, F0827, F0795 and F0556 primary isolates of FIV. Reverse transcriptase activity in the culture supernatants was measured at 1,4 and 6 days postinfection. (D) Luciferase activity in MCC-CON or MCC-CD134 infected with HIV (FIV) luciferase pseudotypes bearing the GL8 or TM2 Envs (mean +/-SE, n=4).

Fig. 3. CD134 expression renders AH927 cells permissive for syncytia formation mediated by FIV Env. FeLV-CR-expressing cells (A-D) or CD134-expressing cells 
(E-H) were transfected with pVR1012 expressing the GL8 (A, E), F0425 (B, F), F0827 (C, G) or PET (D, H) env genes.

Fig. 4. CD134-mediated infection is species specific and CXCR4-dependent. (A) HeLa cells transduced with retroviral vectors bearing feline or human CD134 cDNAs, or a vector only control were infected with HIV (FIV) (GL8 or TM2) or HIV (VSV) luciferase pseudotypes (mean +/-SE, n=4). (B) CD134-dependent infection of HeLa cells expressing feline CD134 by HIV (FIV) pseudotypes bearing a GFP marker gene. CD134 and GFP expression were analyzed by flow cytometry. (C) HeLa cells expressing feline CD134 were infected with HIV (FIV) (GL8 or TM2) or HIV (VSV) luciferase pseudotypes in the presence of 0, 10, 100 or $1000 \mathrm{ng} / \mathrm{ml} \mathrm{CXCR4-antagonist}$ AMD3100 (mean +/-SE, n=4). 


\section{A}

human 1 ..MCVGARRLGRGPCAALLLLGLGLSTVTGLHCVGDTYPSNDRCCHECRP feline 1 MRVVVGAQRP.RAPHSAVQLLGLVLGTAAALHCVGNTYPKDGKCCSECPP

human 49 GNGMVSRCSRSQNTVCRPCGPGFYNDVVSSKPCKPCTWCNLRSGSERKQL feline 50 GYGMESRCSGDQDTKCLQCASGFYNEAVNYEPCKPCTQCNQRSGSEPKQR

human 99 CTATQDTVCRCRAGTQPLDSYKPGVDCAPCPPGHFSPGDNQACKPWTNCT feline 100 CTPTQDTVCRCRPGTEPQDGYDRGVDCAPCPPGHFSPGDDQACKPWTNCT

human 149 LAGKHTLQPASNSSDAICEDRDPPATQPQETQGPPARPITVQPTEAWPRT feline 150 LAGKRTLRPASQGSDAVCEDRSPPATTPWETQGPPVRPPTTQPTTAWPRT

human 199 SQGPSTRPVEVPGGRAVAAILGLGLVLGLLGPLAILLALYLLRRDQRLPP feline 200 SQEPFTPPAEPPRGPQLAAVLGLG. . LGLLAPVAAALALLLHHRAWRLPP human 249 DAHKPPGGGSFRTPIQEEQADAHSTLAKI 277 feline 248 .....GGNSFRTPIQEEHADANSTLAKI 270

B

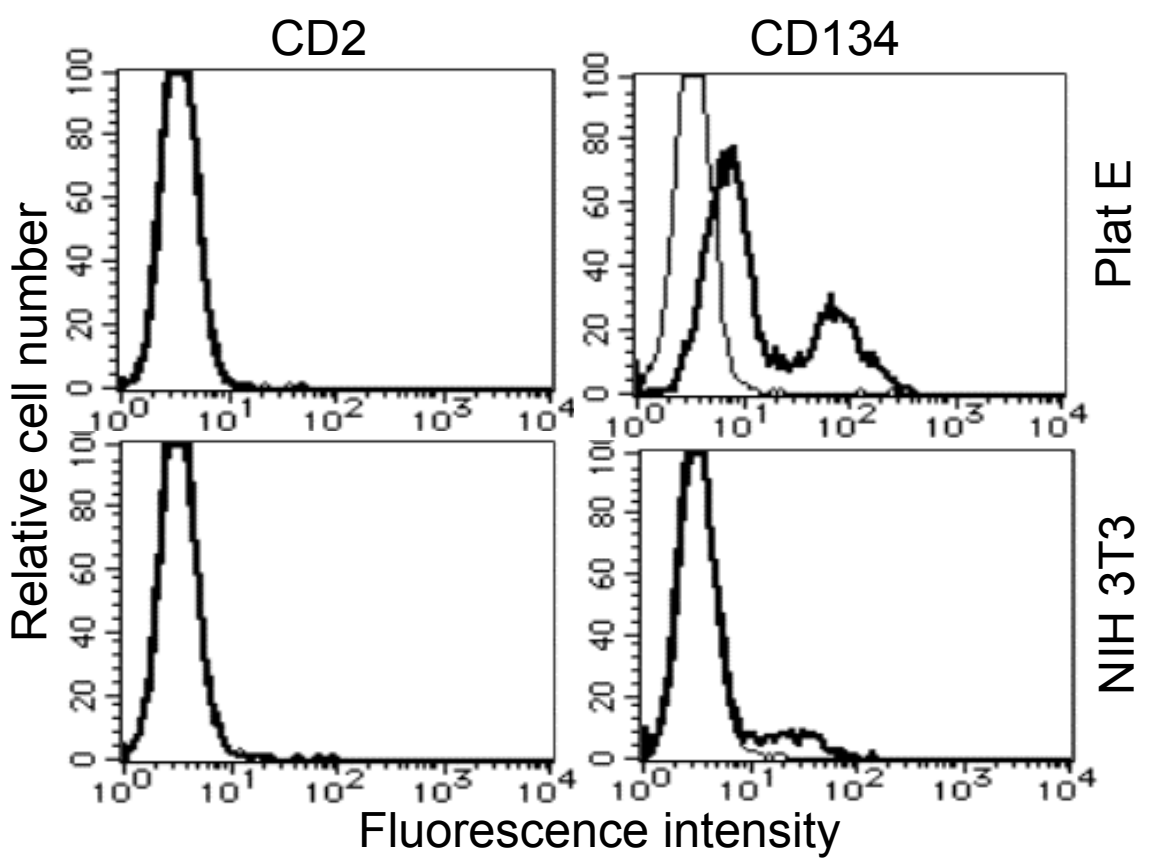


A
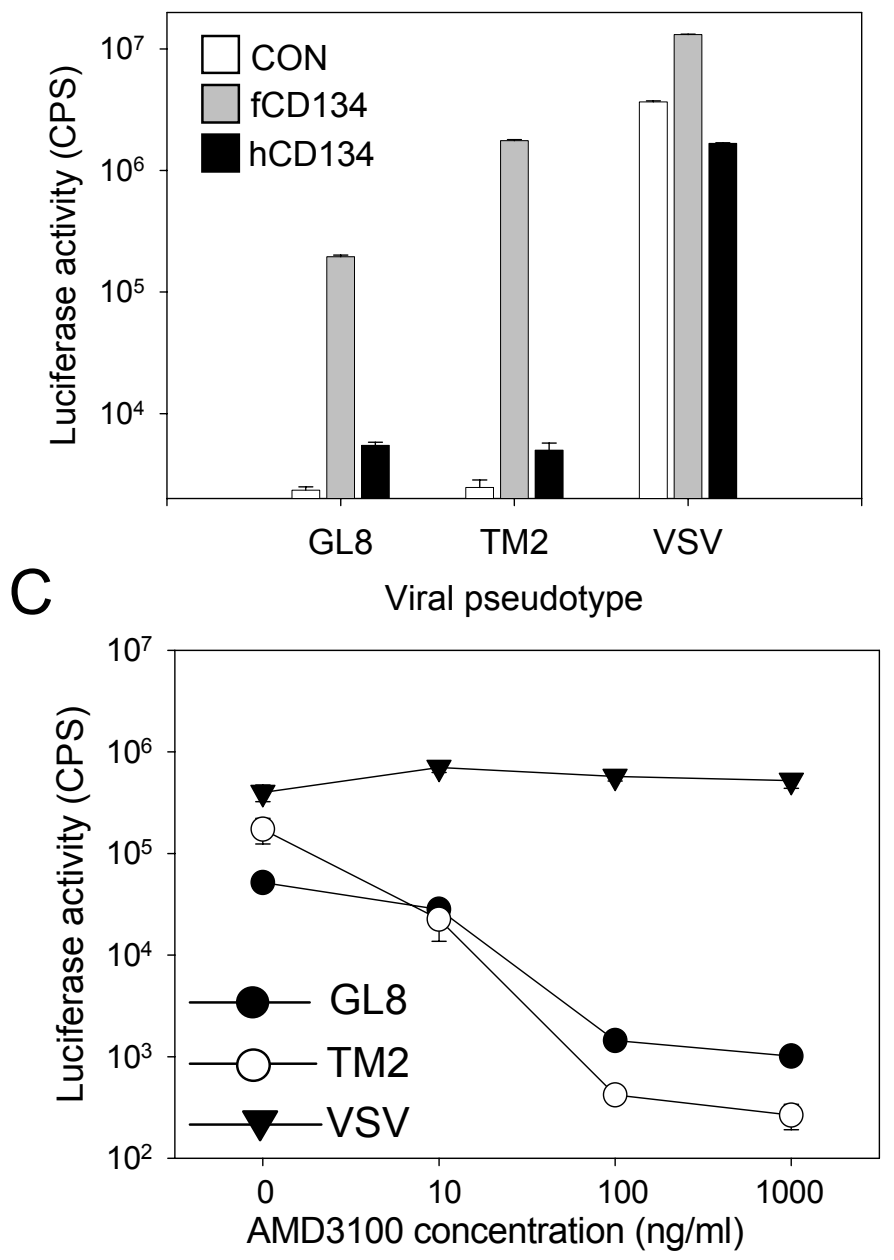

B
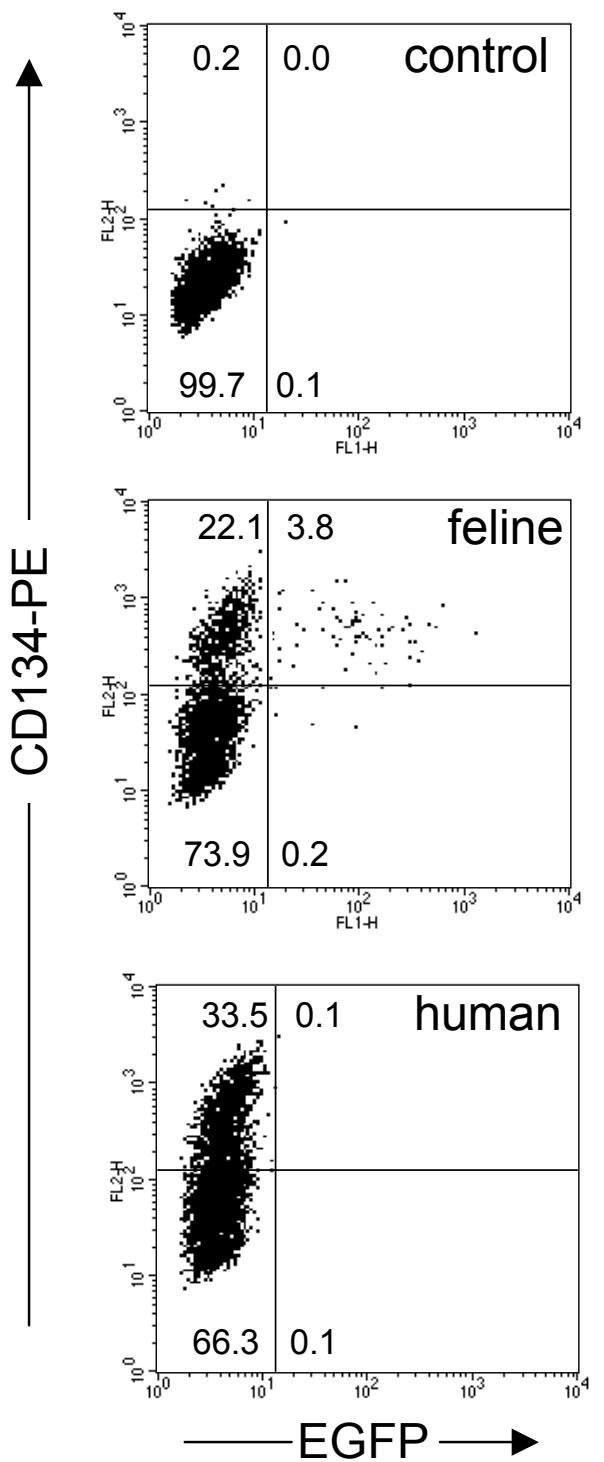

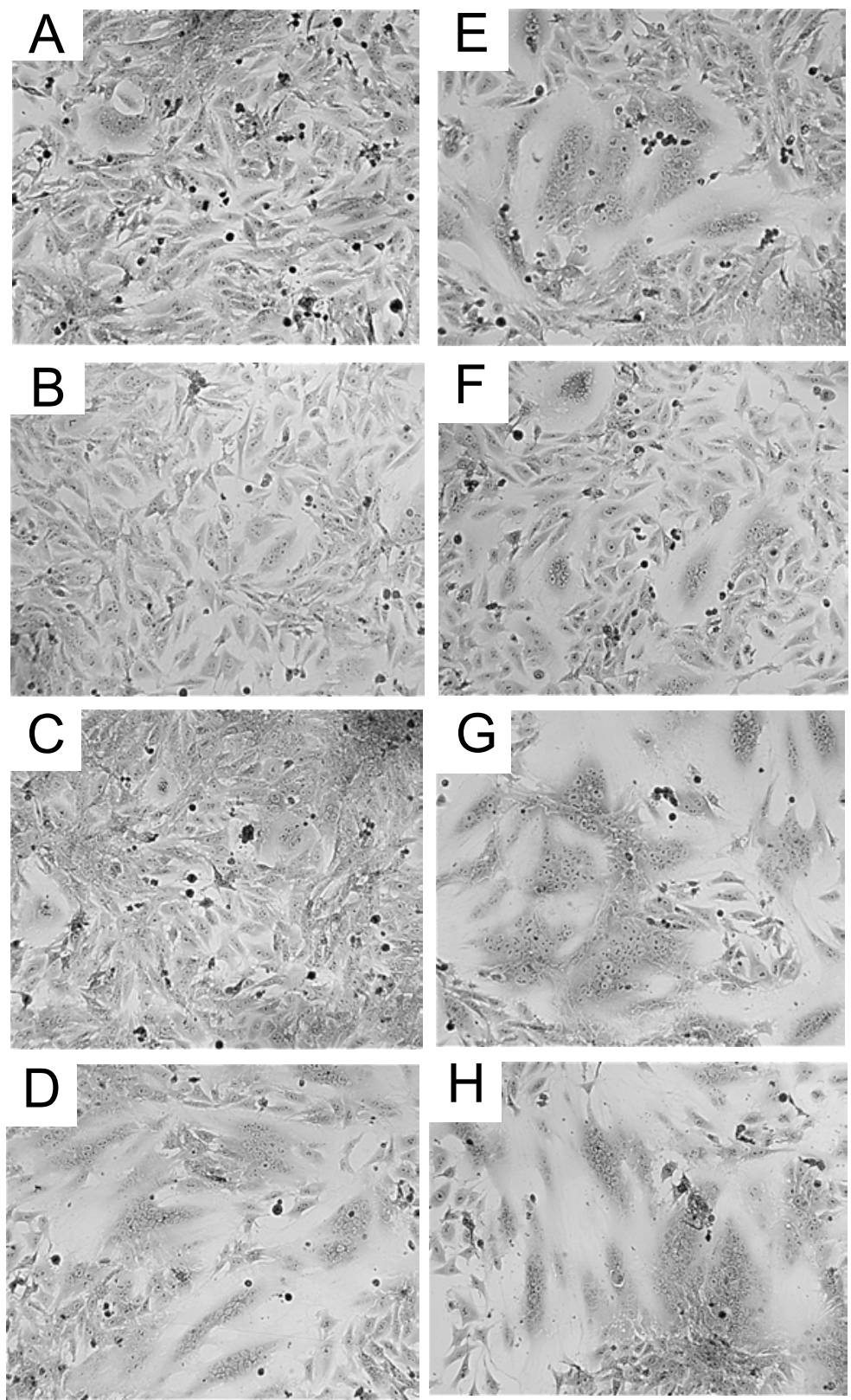
A

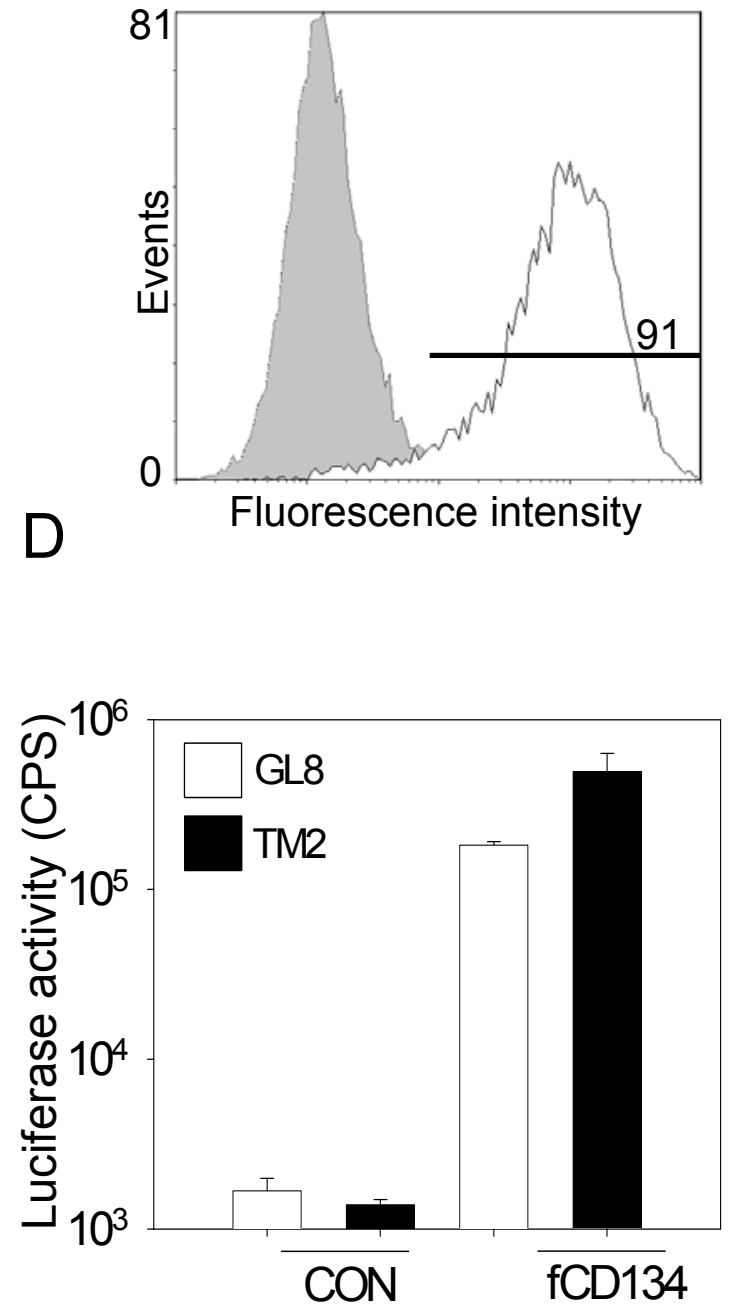

B

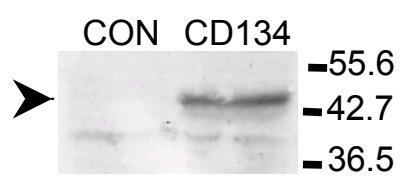

C

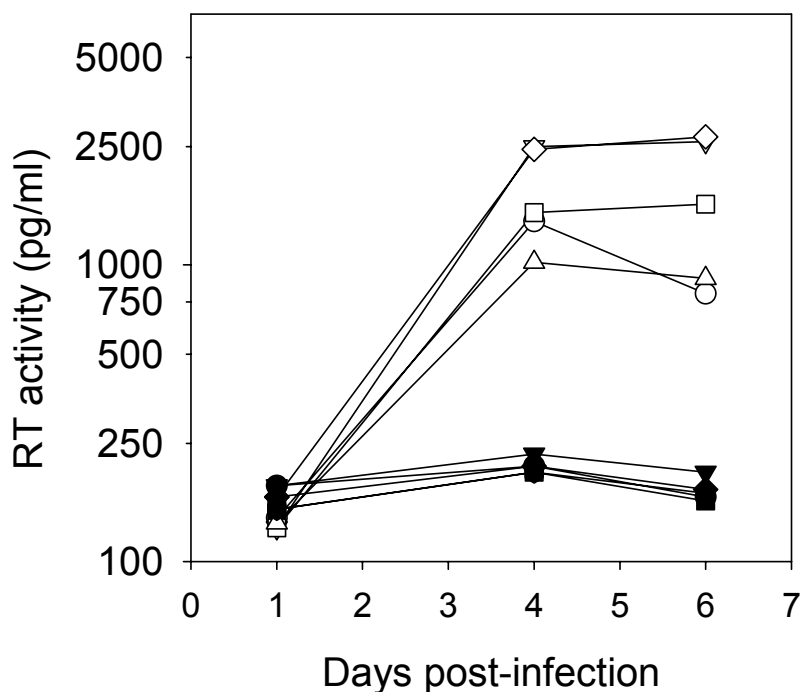

Days post-infection

- CON GL8 -O- fCD134 GL8

CON F0425 $\rightarrow-$ fCD134 F0425

CON F0827 $\square-$ fCD134 F0827

$\checkmark$ CON F0795 $\sim$ fCD134 F0795

$\smile$ CON F0556 $\triangle$ fCD134 F0556 